\title{
Actively Tunable and Polarization-independent Toroidal Resonance in Hybrid Metal-Vanadium Dioxide Metamaterial
}

chang shu ( $\sim$ sum157@163.com )

Harbin University https://orcid.org/0000-0002-3401-4684

Chen Zhang

Harbin University

Yulong Ye

Harbin University

Tongqing Lin

Harbin University

\section{Research Article}

Keywords: Toroidal resonance, Metamaterial, Vanadium dioxide, Actively tunable, Polarizationindependent

Posted Date: November 11th, 2021

DOI: https://doi.org/10.21203/rs.3.rs-987522/v1

License: (c) (i) This work is licensed under a Creative Commons Attribution 4.0 International License. Read Full License 


\title{
Actively tunable and polarization-independent toroidal resonance in hybrid metal-vanadium dioxide metamaterial
}

\author{
Chang Shu ${ }^{1 *}$, Chen Zhang ${ }^{1}$, Yulong Ye ${ }^{1}$, Tongqing Lin ${ }^{1}$ \\ ${ }^{1}$ School of Technology, Harbin University, Harbin, 150086, China \\ *Corresponding author. E-mail:cshu@hrbu.edu.cn
}

\begin{abstract}
Actively tunable and polarization-independent toroidal resonance in hybrid metal-vanadium dioxide metamaterial is proposed and demonstrated numerically in terahertz regime. Simulation results illustrate that a toroidal dipolar resonance is excited by hybrid metal and vanadium dioxide resonator and insensitive with polarization angle of incident plane wave, calculated scattered powers verify the toroidal resonance is strengthened. A novel modulation of resonance strength in proposed toroidal metamaterial is obtained as the phase transition process of vanadium dioxide and contrary to former hybrid metal-vanadium dioxide toroidal metamaterials. The theoretical fitting results reveal that physical mechanism of active modulation in resonance strength can be attributed to the variation of overall damping rate caused by tuning conductivity of vanadium dioxide.
\end{abstract}

Keywords: Toroidal resonance; Metamaterial; Vanadium dioxide; Actively tunable; Polarization-independent.

\section{Introduction}

Toroidal resonance, as a counterpart resonance phenomenon of the fundamental electric and magnetic resonance, was first reported by Zel'dovich in 1957 [1]. In toroidal resonance phenomena, toroidal dipolar resonance can be produced by two reversed closed loops poloidal currents and discovered in nuclear physics and solid state physics [2], which has unique electromagnetic characteristics, such as higher Q-factor and circular dichroism [3]. Unfortunately, toroidal dipolar resonance in natural materials is always accompanied by electric and magnetic resonance, and its electromagnetic scattering power is usually masked by electric and magnetic dipoles [3], which lead to applications of toroidal resonance face difficulties. Therefore, the studies of toroidal response come to standstill for a long time. Recently, metamaterial, which is composed of artificially sub-wavelength resonators, have achieved many unusual characteristics unattainable in natural materials and regarded as an emerging field for extensive exploration of novel electromagnetic devices [4-11]. In 2010, the 
toroidal dipolar response in microwave regime was first experimentally demonstrated with 3D metamaterial [12], in which toroidal dipolar resonance is remarkably strengthened and higher than other multipoles. Consequently, toroidal resonance in metamaterials have attracted enormous attention and considered to unveil a new perspective in toroidal resonance applications [13-19].

Currently, actively tunable electromagnetic characteristics in metamaterial have been drawn considerable attentions for practical requirement, and various kinds of approaches are proposed to achieve actively tunable toroidal resonance phenomenon [20-26]. In these approaches, phase change materials, as efficient methods, have been incorporated into metamaterials to achieve active manipulation of toroidal resonance as the phase transition process. For example, Zhou et al. proposed a Si array and $\mathrm{Ge}_{2} \mathrm{Sb}_{2} \mathrm{Te}_{5}$ (GST) hybrid metamaterial and investigated the radiation manipulation of toroidal resonance via tuning the crystallization states of the GST [26]. In addition, vanadium dioxide $\left(\mathrm{VO}_{2}\right)$ as a representative phase change material shows an excellent transition behavior from insulator phase to metal phase triggering by optical excitation, temperature control and electric fields. Therefore, $\mathrm{VO}_{2}$ has been incorporated into toroidal resonance metamaterial for active manipulation of electromagnetic characteristics. For example, Song et al. proposed $\mathrm{VO}_{2}$ layer beneath the Au-based toroidal resonance structures and achieved active modulation of toroidal dipolar resonance by tuning conductivity of $\mathrm{VO}_{2}[27,28]$. However, these hybrid metal- $\mathrm{VO}_{2}$ toroidal resonance metamaterials are mainly concentrated on $\mathrm{VO}_{2}$ is acting as dielectric layer, and toroidal resonance is polarization sensitive, while little approaches are employed to demonstrate polarization-independent toroidal metamaterial, and toroidal resonator based on metal and $\mathrm{VO}_{2}$ hybrid structure is rarely reported to the present.

In this paper, a polarization-independent toroidal resonance in hybrid metal- $\mathrm{VO}_{2}$ metamaterial is proposed, and the transmission spectral could be actively controlled via phase transition of $\mathrm{VO}_{2}$. Different from former hybrid metal- $\mathrm{VO}_{2}$ metamaterial, the proposed toroidal dipolar phenomenon is excited by metal and $\mathrm{VO}_{2}$ hybrid resonator. Therefore, this work can enrich the actively tunable toroidal dipolar metamaterial and the potential applications in terahertz higher Q-factor sensors.

\section{Structure design and simulation}

The proposed hybrid metamaterial is a periodical structure includes interconnected four $\mathrm{VO}_{2}$ strips and an aluminum-based rectangular ring with four pairs of symmetrical split gaps, which is transferred on $\mathrm{SiO}_{2}$ substrate, as shown in Fig. 1a. The emergency of split gaps can restrain accompaniment of electric and magnetic resonance in the vicinity of toroidal resonance. The dimensions of periodical unit cell are $P_{x}=150 \mu \mathrm{m}$ and $P_{y}=150 \mu \mathrm{m}$ in $x$ and $y$ directions respectively, as shown in Fig. 1b. The geometric parameters of rectangular ring are $L=110 \mu \mathrm{m}, W=10 \mu \mathrm{m}$, $W_{l}=28 \mu \mathrm{m}$, and $g_{l}=2 \mu \mathrm{m}$. The length and width of $\mathrm{VO}_{2}$ strips are $L_{l}=30 \mu \mathrm{m}$ and $W=10$ $\mu \mathrm{m}$ respectively. The thickness of aluminum-based rectangular ring, $\mathrm{VO}_{2}$ strips and $\mathrm{SiO}_{2}$ substrate are same $(10 \mu \mathrm{m})$.

To verify the resonance behaviors of proposed toroidal dipolar metamaterial, 
electromagnetic simulation based on the commercial CST Microwave Studio package is used, in which the $x$ and $y$ axis are unit cell boundary and $\mathrm{z}$ axis is perfect match layer. The incident plane wave with a polarization angle ( $\phi$, shown in Fig. 1b) is normally incident on the $x$ and $y$ axis. The optical characteristics of $\mathrm{VO}_{2}$ in terahertz regime can be expressed by Drude model as $\varepsilon(\omega)=\varepsilon_{\infty}-\frac{\omega_{p}^{2}(\sigma)}{\omega^{2}+i \gamma_{\mathrm{V}_{2}} \omega}$, where $\varepsilon_{\infty}$ and $\gamma_{\mathrm{VO}_{2}}$ are 12 and $5.75 \times 10^{13} \mathrm{rad} / \mathrm{s}$ respectively. The plasma frequency $\omega_{p}$ can be expressed by $\omega_{p}^{2}(\sigma)=\frac{\sigma}{\sigma_{0}} \omega_{p}^{2}\left(\sigma_{0}\right)$ with $\sigma_{0}=3 \times 10^{3} \Omega^{-1} \mathrm{~cm}^{-1}$ and $\omega_{p}\left(\sigma_{0}\right)=1.4 \times 10^{15} \mathrm{rad} / \mathrm{s}$. In this paper, the conductivities of $\mathrm{VO}_{2}(\sigma)$ are analyzed from $1 \times 10^{3} \mathrm{~s} / \mathrm{m}$ to $2 \times 10^{5} \mathrm{~s} / \mathrm{m}$ [29]. The permittivity of $\mathrm{SiO}_{2}$ substrate is taken as 3.9, while permittivity of aluminum can be derived by Drude model with plasma frequency $\omega_{p_{\mathrm{AL}}}=2.24 \times 10^{16} \mathrm{rad} / \mathrm{s}$ and the damping constant $\gamma_{\mathrm{Al}}=1.22 \times 10^{14} \mathrm{rad} / \mathrm{s}$ [30].

\section{Results and discussion}

Due to the symmetrical characteristic, the proposed metamaterial exhibit the identical response to incident waves with arbitrarily polarization angle. When the initial conductivity of $\mathrm{VO}_{2}(\sigma)$ is $2 \times 10^{5} \mathrm{~s} / \mathrm{m}$, the transmission spectrum of proposed metamaterial as the polarization angle $\phi$ varying from $0^{\circ}$ to $90^{\circ}$ are shown in Fig. 2. As observed in Fig. 2, there are perfect polarization-independent properties and obviously sharp resonance dip at $1.45 \mathrm{THz}$ with quality factor Q is 96.4. Here, quality factor $\mathrm{Q}$ is defined as ratio of central frequency to resonant linewidth [31].

In order to understand forming mechanism of toroidal dipolar resonance, the electric fields, surface currents and magnetic fields distributions of hybrid resonator at $1.45 \mathrm{THz}$ for different polarization angle $\phi$ are displayed. As observed from Fig. 3a-c, when polarization angle $\phi$ is $0^{\circ}$, a pair of opposite electric charges emergence in neighboring edges of horizontal $\mathrm{VO}_{2}$ strips, and two reversed closed loops poloidal currents are produced and distributed in quarter I, II hybrid section and quarter III, IV hybrid section, as a result, magnetic dipoles with head-to-tail arrangement are produced and a toroidal dipolar resonance along the x-direction is excited. This forming mechanism of toroidal dipolar resonance is more clearly seen in Fig. 3d.

On the contrary, As observed from Fig. 4a-c, when polarization angle $\phi$ is $90^{\circ}$, a pair of opposite electric charges emergence in neighboring edges of vertical $\mathrm{VO}_{2}$ strips, and two reversed closed loops poloidal currents are produced and distributed in quarter I, IV hybrid section and quarter II, III hybrid section, as a result, magnetic dipoles with head-to-tail arrangement are produced and a toroidal dipolar resonance along the y-direction is excited. This forming mechanism of toroidal dipolar resonance is more clearly seen in Fig. 4d.

In addition, the forming mechanism of toroidal dipolar resonance when polarization angle $\phi$ is $45^{\circ}$ has also been studied and demonstrated in Fig. 5. As observed from Fig. 5a, magnetic dipoles with head-to-tail arrangement are distributed in diagonal sections and a toroidal dipolar resonance along $45^{\circ}$ angle with $\mathrm{x}$-direction is excited. This forming mechanism of toroidal dipolar resonance is more clearly seen in Fig. $5 b$.

As we mentioned above, toroidal resonance in natural materials is always mas ked by electric and magnetic dipoles, and application of metamaterial could 
strengthen its contribution. To quantitatively analyze this contribution, the scattered powers excited by proposed metamaterial are calculated through current density displacement in unit cell [32,33]. Fig. 6 shows the calculated scattered powers of conventional multipoles and toroidal dipole in proposed metamaterial as the conductivity of $\mathrm{VO}_{2}$ is $2 \times 10^{5} \mathrm{~s} / \mathrm{m}$ and polarization angle $\phi$ of incident plane wave is $0^{\circ}$, which includes five strongest powers: electric dipole $P_{y}$, magnetic dipole $M_{z}$, toroidal dipole $T_{y}$, electric quadrupole $Q_{e}$ and magnetic quadrupole $Q_{m}$. As observed from Fig. 6 , in the vicinity of $1.45 \mathrm{THz}$, the electric dipole $P_{y}$ is strongly suppressed while the scattered power of toroidal dipolar resonance increases remarkably and plays a dominant role. Therefore, these results indicate that the transmission spectra resonate at $1.45 \mathrm{THz}$ is mainly attributed to toroidal dipolar resonance.

Next, because of $\mathrm{VO}_{2}$ has been incorporated into resonator, the proposed toroidal dipolar phenomena could be actively modulated. Fig. 7 shows the transmission spectra of the toroidal dipolar metamaterial in the case of varying the conductivity of $\mathrm{VO}_{2}(\sigma)$ when polarization angle $\phi$ of incident plane wave is $0^{\circ}$.

As shown in Fig. 7, the frequency of toroidal resonance exhibits slightly red shift and amplitude decreases as conductivity of $\mathrm{VO}_{2}(\sigma)$ decreases, specifically, the toroidal resonance is disappeared as $\sigma$ is $1000 \mathrm{~s} / \mathrm{m}$. It should be pointed out that this tuning characteristic of resonance amplitude in proposed toroidal dipolar metamaterial as conductivity of $\mathrm{VO}_{2}(\sigma)$ decreases is contrary to former hybrid metal- $\mathrm{VO}_{2}$ toroidal metamaterial $[27,28]$. While the frequency red shift phenomenon can be attributed to the increase of resistance in SRRs, which can be explained by resonance frequency expression: $f=\frac{1}{2 \pi}\left(\frac{1}{L C}-\frac{R^{2}}{4 L^{2}}\right)^{\frac{1}{2}}[34]$, here, $L$ is effective inductance, $C$ is effective capacitance, and $R$ is resistance in SRRs.

As well known, the transmission feature of toroidal resonance metamaterial could be fitted by Fano formula [35,36]:

$$
T=\left|a_{1}+i a_{2}+\frac{b}{\omega-\omega_{0}+i \gamma}\right|^{2}
$$

Where $a_{1}, a_{2}$ and $b$ are constant, $\gamma$ is the damping rate, and $\omega_{0}$ is the resonant frequency. To further reveal physical mechanism of active modulation in case of varying the conductivity of $\mathrm{VO}_{2}$ when polarization angle $\phi$ of incident plane wave is $0^{\circ}$, the fitted transmission spectra are calculated and shown in Fig. 8. It is obviously observed that the fitted curves exhibit excellent agreement with the simulated curves. Fig. 9 show the damping rates of proposed metamaterial in case of varying the conductivity of $\mathrm{VO}_{2}$ when polarization angle $\phi$ of incident plane wave is $0^{\circ}$. As shown in Fig. 9, the overall damping rate $\gamma$ increases remarkably as conductivity of $\mathrm{VO}_{2}$ decreases, which is consistent with feature of the proposed toroidal dipolar phenomenon is excited by metal and $\mathrm{VO}_{2}$ hybrid resonator.

\section{Conclusions}

In conclusions, we have numerically demonstrated an actively tunable and polarization-independent toroidal dipolar resonance analogue based on hybrid metal- $\mathrm{VO}_{2}$ metamaterial. The proposed hybrid metamaterial consists of interconnected $\mathrm{VO}_{2}$ strips and an aluminum-based rectangular ring with four pairs of symmetrical split gaps. Different from former hybrid metal- $\mathrm{VO}_{2}$ metamaterial, the proposed toroidal dipolar phenomenon is excited by metal and $\mathrm{VO}_{2}$ hybrid resonator. 
Simulation results illustrate two reversed closed loops poloidal currents are produced to excite a toroidal dipolar resonance, which exhibit insensitive with polarization angle of incident plane wave. The calculated scattered powers verify the toroidal dipolar resonance increases remarkably and plays a dominant role in the vicinity of transmission dip. In addition, active modulation of toroidal dipolar resonance is manifested via changing conductivity of $\mathrm{VO}_{2}$, and the amplitude of toroidal resonance decreases and frequency exhibits slightly red shift as conductivity of $\mathrm{VO}_{2}$ decreases, specifically, the toroidal resonance is disappeared as conductivity of $\mathrm{VO}_{2}$ is $1000 \mathrm{~s} / \mathrm{m}$. The theoretical fitting results reveal that physical mechanism of active modulation in resonance amplitude can be attributed to the variation of overall damping rate caused by tuning conductivity of $\mathrm{VO}_{2}$. Therefore, this work can enrich the actively tunable toroidal dipolar metamaterial and the potential applications in terahertz higher Q-factor sensors.

Author Contribution Methodology and writing - original draft preparation, Chang Shu; software and figure, Chen Zhang and Yulong Ye; review and editing, Tongqing Lin. All authors have read and agreed to the published version of the manuscript.

Funding The work is supported by Harbin University Doctor Found (HUDF2020108), and Innovation and Entrepreneurship Training Program for College Students in Heilongjiang Province (202110234035).

Data Availability The data that support the findings of this study are available from the corresponding author upon reasonable request.

Code Availability Not applicable.

Ethics approval Not applicable.

\section{Declarations}

Consent to Participate All authors agreed to participate in this research study.

Consent to Publish Permission from all the authors has been taken to publish this manuscript.

Conflict of Interest The authors declare that they have no conflict of interest.

\section{References}

[1] Zeldovich IB (1958) Electromagnetic interaction with parity violation. Sov. Phys. JETP. 6: 1184-1186

[2] Dubovik VM, Tugushev VV (1990) Toroid moments in electrodynamics and solid-state. physics Phys. Rep. 187:145 
[3] Li MH, Guo LY, Dong JF, Yang HL (2014) Resonant transparency in planar metamaterial with toroidal moment. Appl.Phys. Express 7: 082201

[4] Zhou J, Zhang L, Tuttle G, Koschny T, Soukoulis CM (2006) Negative index materials using simple short wire pairs. Phys. Rev. B 73: 041101

[5] Zharov AA, Shadrivov IV, Kivshar YS (2003) Nonlinear properties of left-handed metamaterials. Phys. Rev. Lett. 91: 037401

[6] Landy NI, Sajuyigbe S, Mock JJ, Smith DR, Padilla WJ (2008) Perfect metamaterial absorber. Phys. Rev. Lett. 100: 207402

[7] Luo C, Ibanescu M, Johnson SG, Joannopoulos J (2003) Cerenkov radiation in photonic crystals. Science 299: 368-371

[8] Seddon N, Bearpark T (2003) Observation of the inverse Doppler effect. Science 302: 1537-1540

[9] Mei JS, Wu Q, Zhang K (2012) Multifunctional complementary cloak with homogeneous anisotropic material parameters. J. Opt. Soc. Am. A 29: 2067-2073

[10] Pendry JB (2000) Negative refraction makes a perfect lens. Phys. Rev. Lett. 85: 3966

[11] Li J, Yu P, Cheng H, Liu W, Li Z, Xie B, Chen S, Tian J (2016) Optical polarization encoding using graphene-loaded plasmonic metasurfaces. Adv. Opt. Mater. 4 :91-98

[12] Kaelberer T, Fedotov VA, Papasimakis N, Tsai DP, Zheludev N I (2010) Toroidal dipolar response in a metamaterial. Science 330: 1510-1512

[13] Xu S, Sayanskiy A, Kupriianov AS, Tuz VR, Kapitanova P, Sun HB (2018) Experimental observation of toroidal dipole modes in all-dielectric metasurfaces. Adv. Opt. Mater. 1801166

[14] Liu Z, Du S, Cui A, Li ZC, Fan YC, Chen SQ (2017) High-quality-factor mid-infrared toroidal excitation in folded 3D metamaterials. Adv. Mater. 1606298

[15] Huang YW, Chen WT, Wu PC, Fedotov V, Savinov V, Ho ZY (2012) Design of plasmonic toroidal metamaterials at optical frequencies. Opt. Express 20: 1760

[16] Fan Y, Wei Z, Li H, Chen H, Soukoulis CM (2013) Low-loss and high-Q planar metamaterial with toroidal moment. Phys. Rev. B 87: 115417

[17] Dong ZG, Ni P, Zhu J, Yin X, Zhang X (2012) Toroidal dipole response in a multifold double-ring metamaterial. Opt. Express 20: 13065

[18] Fedotov VA, Rogacheva AV, Savinov V, Tsai DP, Zheludev NI (2013) Resonant transparency and non-trivial non-radiating excitations in toroidal metamaterials. Sci. Rep. 3: 2967

[19] Guo LY, Li MH, Huang XJ, Yang HL (2014) Electric toroidal metamaterial for resonant transparency and circular cross-polarization conversion. Appl. Phys. Lett. 105: 033507

[20] Gerislioglu B, Ahmadivand A, Pala N (2018) Tunable plasmonic toroidal terahertz metamodulator. Phys. Rev. B 97: 161405

[21] Gupta M, Srivastava YK, Singh R (2018) A toroidal metamaterial switch. Adv. Mater. 30: 1704845

[22] He X, Tian L, Wang Y, Jiang J, Geng Z (2020) Active modulation and switching of toroidal resonance in micromachined reconfigurable terahertz metamaterials. Results Phys. 17: 103133

[23] Sun Y, Liao D, Xu J, Wu Y, Chen L (2020) Active switching of toroidal resonances by using a Dirac semimetal for terahertz communication. Front Phys. 8: 602772

[24] Chen X, Fan W (2017) Study of the Interaction between Graphene and Planar Terahertz 
Metamaterial with Toroidal Dipolar Resonance. Opt. Lett. 42: 2034-2037

[25] Liu Z, Du S, Cui A, Li Z, Fan Y, Chen S, Li W, Li J, Gu C (2017) High-quality-factor mid-infrared toroidal excitation in folded 3D metamaterials. Adv. Mater. 29: 1606298

[26] Zhou CB, Li SY, Fan MH, Wang XF, Xu YL, Xu WW, Xiao SY, Hu MZ, Liu JT (2020) Optical radiation manipulation of Si-Ge2Sb2Te5 hybrid metasurfaces. Opt. Express 28: 9690-9701

[27] Song Z, Deng Y, Zhou Y, Liu Z (2019) Tunable toroidal dipolar resonance for terahertz wave enabled by a vanadium dioxide metamaterial. IEEE Photonics J. 11: 1-5

[28] Song Z, Deng Y, Zhou Y, Liu Z (2019) Terahertz toroidal metamaterial with tunable properties. Opt. Express 27: 5792-5797

[29] Huang J, Li J, Yang Y, Li J, Li JH, Zhang YT, Yao JQ (2020) Broadband terahertz absorber with a flexible, reconfigurable performance based hybrid-patterned vanadium dioxide metasurfaces. Opt. Express 28: 17832-17840

[30] Xiao S, Wang T, Liu T, Yan X, Li Z, Xu C (2018) Active modulation of electromagnetically induced transparency analogue in terahertz hybrid metal-graphene metamaterials. Carbon 126: $271-278$

[31] Malthesh S, Krishnaswamy N (2019) Improvement in quality factor of double microring resonator for sensing applications. J. Nanophotonics 13: 026014

[32] Sun G, Peng S, Zhang X, Zhu Y (2020) Switchable Electromagnetically Induced Transparency with Toroidal Mode in a Graphene-Loaded All-Dielectric Metasurface. Nanomaterials 10: 1064

[33] Liu GD, Zhai X, Xia SX, Lin Q, Zhao CJ, Wang LL (2017) Toroidal resonance based optical modulator employing hybrid graphene-dielectric metasurface. Opt. Express 25: 26045-26054

[34] Chen HT, Yang H, Singh R, O'Hara JF, Azad AK, Trugman SA, Jia QX, Taylor AJ (2010) Tuning the resonance in high-temperature superconducting terahertz metamaterials. Phys. Rev. Lett. 105: 247402

[35] Wang WD, Qi JG (2019) Polarization-independent Fano metasurface with directional toroidal dipole for magnetic field tunability. Appl. Phys. Express 12: 065004

[36] Bhattacharya A, Devi KM, Nguyen T, Kumar G (2020) Actively tunable toroidal excitations in graphene based terahertz metamaterials. Opt. Commun. 459: 124919 


\section{Figure captions}

Figure 1. Toroidal dipolar metamaterial based on metal-vanadium dioxide: (a) schematic of metamaterial, (b) close-up view of unit cell,

Figure 2. Calculated transmission spectra of toroidal resonance as the polarization angle $\phi$ varying from $0^{\circ}$ to $90^{\circ}$

Figure 3. The electric fields, surface currents and magnetic fields distributions of hybrid resonator at $1.45 \mathrm{THz}$ when polarization angle $\phi$ is $0^{\circ}$ : (a) electric fields, (b) surface currents, (c) magnetic fields, (d) schematic of toroidal dipole excitation in unit cell

Figure 4. The electric fields, surface currents and magnetic fields distributions of hybrid resonator at $1.45 \mathrm{THz}$ when polarization angle $\phi$ is $90^{\circ}$ : (a) electric fields, (b) surface currents, (c) magnetic fields, (d) schematic of toroidal dipole excitation in unit cell

Figure 5. The magnetic fields distributions and toroidal dipole excitation of hybrid resonator at $1.45 \mathrm{THz}$ when polarization angle $\phi$ is $45^{\circ}$ : (a) magnetic fields, (d) schematic of toroidal dipole excitation in unit cell

Figure 6. Scattered powers of conventional multipoles and toroidal dipole in proposed metamaterial when polarization angle $\phi$ is $0^{\circ}$

Figure 7. Transmission spectra of toroidal dipolar metamaterial with conductivity of $\mathrm{VO}_{2}$ is varying

Figure 8. The fitted transmission spectra with conductivity of $\mathrm{VO}_{2}$ is varying

Figure 9. The fitted overall damping rate $\gamma$ with conductivity of $\mathrm{VO}_{2}$ is varying 
Figure list

Figure 1

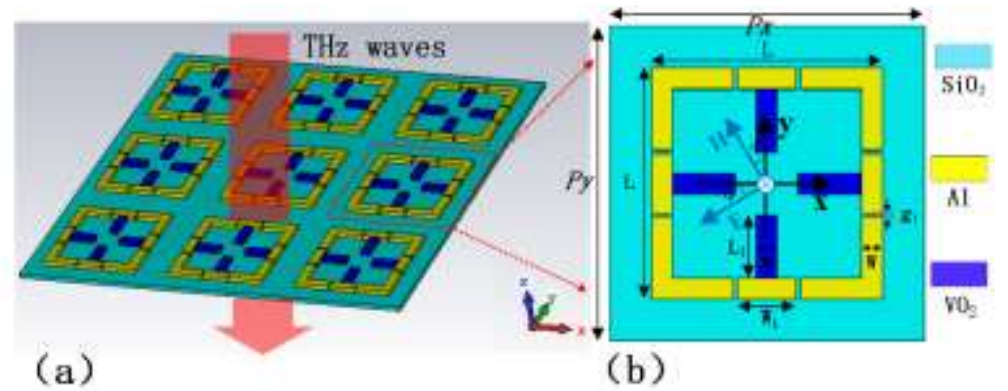

Figure 2

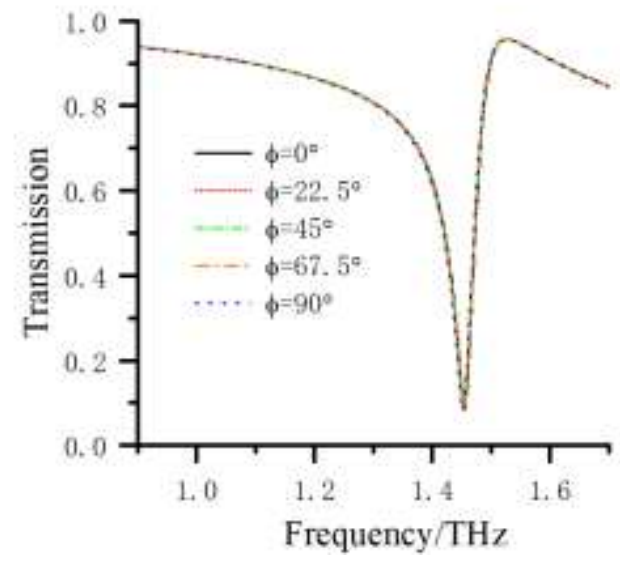

Figure 3

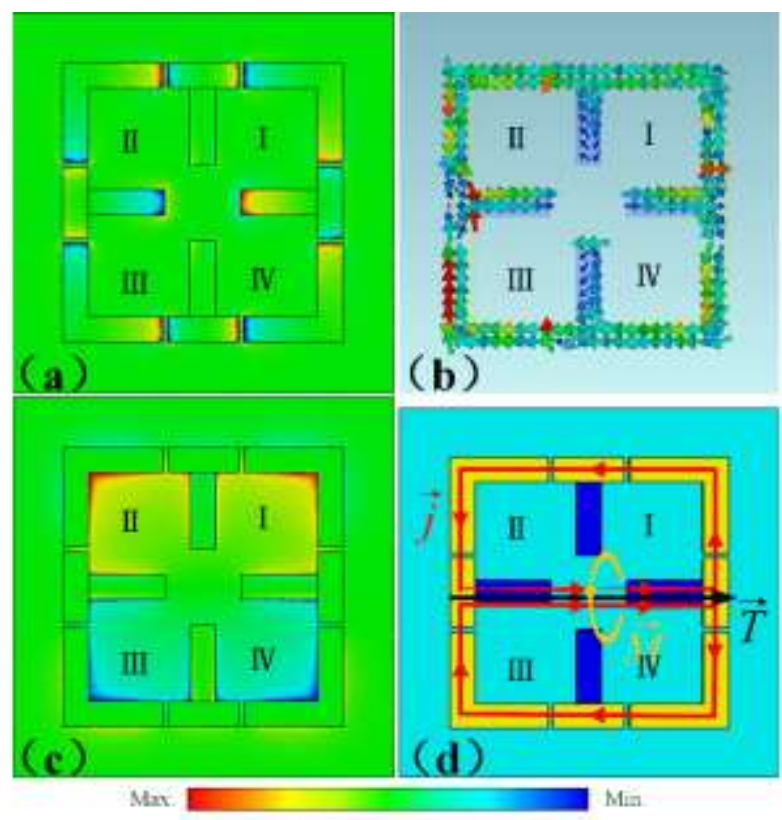


Figure 4

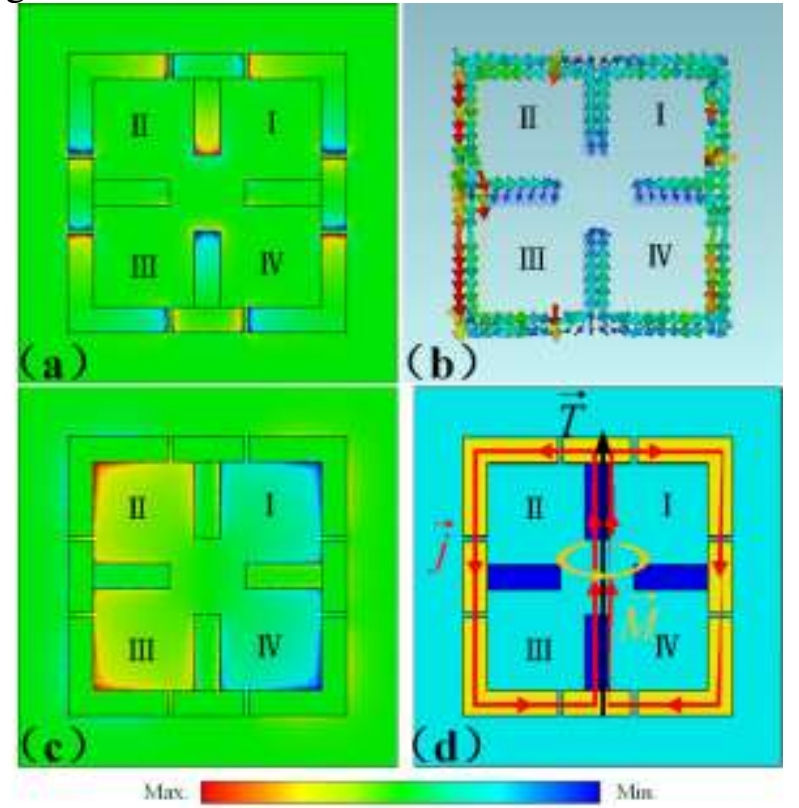

Figure 5

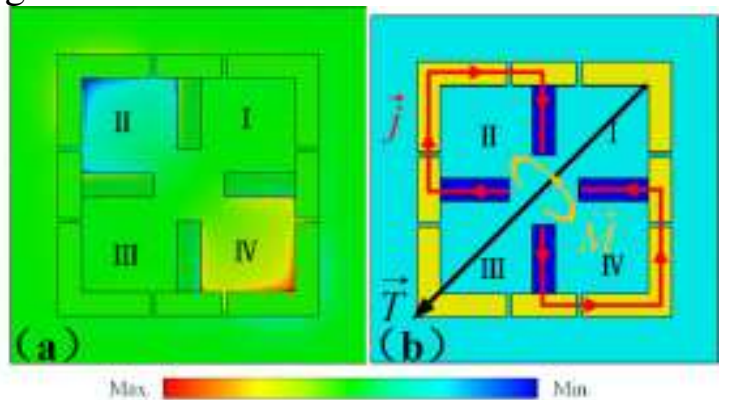

Figure 6

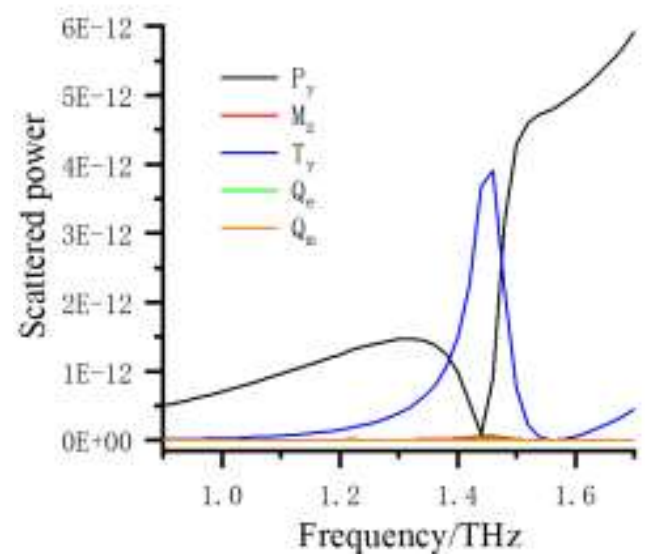


Figure 7

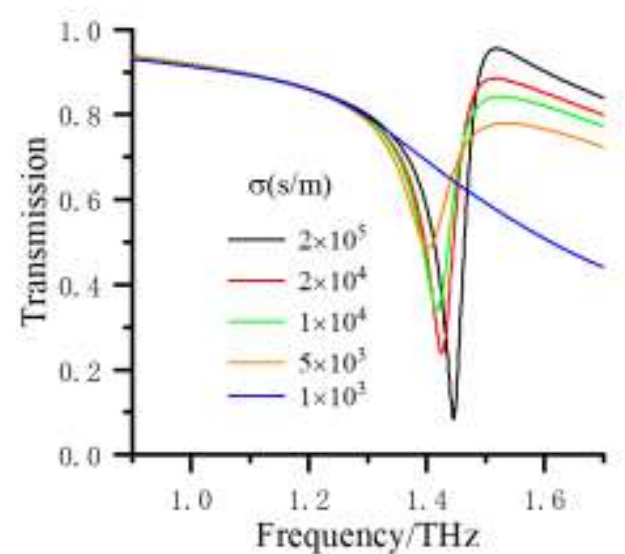

Figure 8

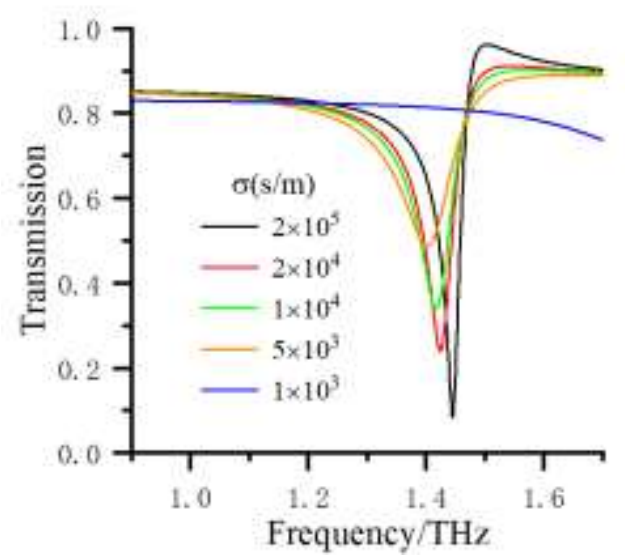

Figure 9

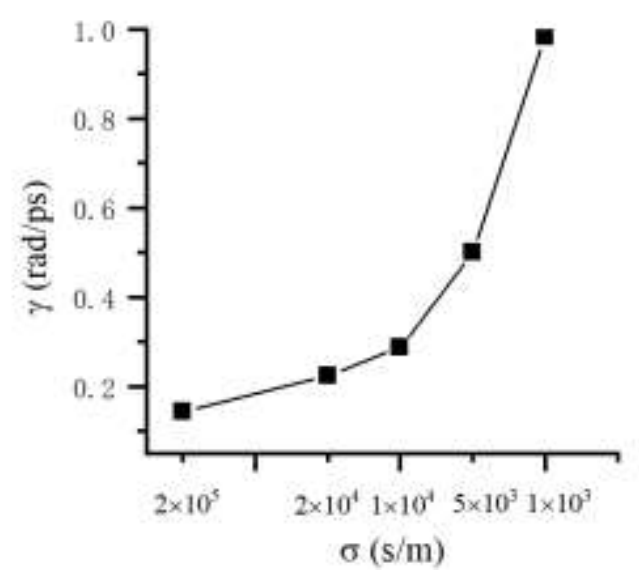

